\title{
Adding an invasive procedure will not necessarily change treatment or outcome of NSCLC patients with preoperative clinical N1 disease
}

To the Editor:

We have read with great interest and welcome the publication of a prospective multicentre study regarding the role of mediastinal staging by video-assisted mediastinoscopy (VAM) in patients with clinical N1 (cN1) nonsmall cell lung cancer (NSCLC) [1]. The objective of the study was to assess the sensitivity, negative predictive value and accuracy of VAM in a well-defined group of patients with $\mathrm{cN} 1$.

This nonrandomised prospective study planned to recruit 250 NSCLC patients with stage cT1-3N1M0 deemed operable based on integrated fluorodeoxyglucose-positron-emission tomography/computed tomography. The surgical resection with systemic nodal dissection was the reference standard. The primary end-point was sensitivity, defined as the proportion of patients with positive mediastinal staging by VAM or VAM-lymphadenectomy (VAMLA) out of all the patients with mediastinal nodal disease.

The authors concluded that $25 \%$ of the patients within the study eventually had unsuspected N2 disease with a $73 \%$ sensitivity by VAM and proposed the procedure as a possible standard of care in this setting.

As conceived by the authors, there are several limitations in this study, two particularly important and worth mentioning further. The first regards the slow and inadequate recruitment leading to only 105 patients being enrolled into the study instead of the pre-planned 250 patients. The second point is the fact that in nearly one-third (31\%) of cases the nodal dissection was performed by VAMLA and not only by VAM. Although similar, the two procedures are different: during VAMLA a systematic lymphadenectomy is performed bi-manually through the video mediastinoscope and the number of lymph nodes removed is doubled compared to standard mediastinoscopy [2].

Although these two issues do not allow us to conclude that VAM(LA) could be a new standard in cN1 NSCLC patients, another point regards the real implication of routinely performing VAM(LA) in this setting. Even if this is recommended within the guidelines of the European Society of Thoracic Surgeons [3], the level of evidence is certainly not that strong (level IIB) and unlikely to change the outcome or treatment of such patients. There is also evidence that patients with unsuspected N2 disease have a better overall survival than those with standard N2 nodal disease [4] and that those with single-station N2 involvement have similar overall survival than those with multiple N1 disease, as established recently [5].

In conclusion, we should certainly praise the authors for making such an effort to design and conduct a multicentre prospective study; however, we believe that there is not adequate evidence to justify VAM(LA) as a standard tool to adopt in all cN1 NSCLC patients preoperatively.

Alfredo Addeo ${ }^{1}$ and Giuseppe Banna ${ }^{2}$

${ }^{1}$ Oncology Dept, University Hospital Geneva, Geneva, Switzerland. ${ }^{2}$ Division of Medical Oncology, Cannizzaro Hospital, Catania, Italy.

Correspondence: Alfredo Addeo, Oncology Dept, University Hospital Geneva, Rue Gabrielle-Perret-Gentil 4, 1205 Geneva, Switzerland. E-mail: alfredo.addeo@hcuge.ch

Received: Jan 152018 | Accepted: Jan 232018

@ERSpublications

Video-assisted mediastinoscopy or VAM-lymphadenectomy is not a standard procedure in cN1 disease http://ow.ly/dAZq30irs63

Cite this article as: Addeo A, Banna G. Adding an invasive procedure will not necessarily change treatment or outcome of NSCLC patients with preoperative clinical N1 disease. Eur Respir J 2018; 51: 1800084 [https://doi.org/10.1183/13993003.00084-2018]. 
Conflict of interest: None declared.

\section{References}

1 Decaluwé $\mathrm{H}$, Dooms C, D'Journo XB, et al. Mediastinal staging by videomediastinoscopy in clinical N1 non-small cell lung cancer: a prospective multicentre study. Eur Respir J 2017; 50: 1701493.

2 Leschber G, Holinka G, Linder A. Video-assisted mediastinoscopic lymphadenectomy (VAMLA) - a method for systematic mediastinal lymphnode dissection. Eur J Cardiothorac Surg 2003; 24: 192-195.

3 De Leyn P, Dooms C, Kuzdzal J, et al. Revised ESTS guidelines for preoperative mediastinal lymph node staging for non-small-cell lung cancer. Eur J Cardiothorac Surg 2014; 45: 787-798.

4 Thomas DC, Arnold BN, Rosen JE, et al. The significance of upfront knowledge of N2 disease in non-small cell lung cancer. World J Surg 2018; 42: 161-171.

5 Asamura H, Chansky K, Crowley J, et al. The International Association for the Study of Lung Cancer Lung Cancer Staging Project: Proposals for the Revision of the N Descriptors in the Forthcoming 8th Edition of the TNM Classification for Lung Cancer. J Thorac Oncol 2015; 10: 1675-1684.

From the authors:

We would like to thank A. Addeo and G. Banna for their correspondence with comments on our article entitled "Mediastinal staging by videomediastinoscopy in clinical N1 non-small cell lung cancer: a prospective multicentre study" [1]. In this prospective multicentre study, we found a $25 \%$ rate of unforeseen N2 disease after staging and resection, confirming the result of a previous prospective study, similar in size and design, investigating the performance of endosonography in the mediastinal staging of clinical N1 (cN1) patients [2]. However, the sensitivity and negative predictive value of videomediastinoscopy versus endosonography was 0.73 (95\% CI $0.54-0.86$ ) versus 0.38 (95\% CI $0.18-0.57$ ) and 0.92 (95\% CI $0.83-0.97$ ) versus 0.81 (95\% CI $0.71-0.91$ ), respectively. We acknowledge the lower accrual than initially projected, which resulted in wider width of confidence interval than aimed for. Nonetheless, with these results we argue that videomediastinoscopy could be the preferred technique of invasive mediastinal staging in patients with $\mathrm{cN} 1$ disease, outperforming endosonography in this patient group.

Whether invasive staging should be performed at all in patients with $\mathrm{cN} 1$ disease is a different point of discussion that was not part of this study. Correct staging prior to the start of therapy is not only responsible for an apparent better survival due to stage migration, but also leads to diverse surgical and non-surgical treatment strategies in individual patients, and is of paramount importance for comparative purposes. Furthermore, invasive staging in patients with $\mathrm{cN1}$ is indeed recommended by the current guidelines of the European Society of Thoracic Surgeons and the European Society for Medical Oncology [3, 4].

We acknowledge that one third of the videomediastinoscopy procedures were video-assisted mediastinoscopic lymphadenectomies (VAMLA), although not all authors use a bi-manual instrumentation as suggested in the correspondence by A. Addeo and G. Banna. We want to stress to the readers that videomediastinoscopy and VAMLA are performed through the same small incision with similar instruments. While VAMLA goes beyond a pure diagnostic procedure and might be a first step in a complete lymphadenectomy, VAMLA should not be confused with transcervical extended mediastinal lymphadenectomy (TEMLA), which is performed through a cervical incision of $5-8 \mathrm{~cm}$ and includes elevation of the sternal manubrium and complete mediastinal lymphadenectomy except for stations 9 and most distal 4L [5]. In our study, the VAMLA results showed no false negatives and no complications [1]. The numbers were too small to compare standard videomediastinoscopy with VAMLA, but in our opinion a pre-resection VAMLA can help to perform a complete mediastinal lymphadenectomy in these $\mathrm{cN} 1$ patients with clearly significant clinical risk of mediastinal nodal disease.

@ERSpublications

In patients with clinical N1 NSCLC, videomediastinoscopy outperforms endosonography as a mediastinal staging tool http://ow.ly/ShP330iWzN2

Cite this article as: Decaluwé $\mathrm{H}$, Dooms C, De Leyn $\mathrm{P}$, et al. Adding an invasive procedure will not necessarily change treatment or outcome of NSCLC patients with preoperative clinical N1 disease. Eur Respir J 2018; 51: 1800410 [https://doi.org/10.1183/13993003.00410-2018]. 
Herbert Decaluwé ${ }^{1}$, Christophe Dooms ${ }^{2}$, Paul De Leyn ${ }^{1}$, Pascal Thomas ${ }^{3}$ and Ramon Rami-Porta ${ }^{4}$

${ }^{1}$ Dept of Thoracic Surgery, University Hospitals Leuven, Leuven, Belgium. ${ }^{2}$ Dept of Pneumology, University Hospitals Leuven, Leuven, Belgium. ${ }^{3}$ Dept of Thoracic Surgery, Lung Transplantation and Diseases of the Esophagus, North University Hospital, Marseille, France. ${ }^{4}$ Dept of Thoracic Surgery, Hospital Universitari Mutua Terrassa, Terrassa, Spain.

Correspondence: Herbert Decaluwé, Herestraat 49, 3000 Leuven, Belgium. E-mail: Herbert.decaluwe@uzleuven.be

Received: Feb 272018 | Accepted: Feb 272018

Conflict of interest: H. Decaluwé reports personal fees from Covidien Medtronic for training lectures, outside the submitted work. P. Thomas reports personal fees from Ethicon and Covidien Medtronic for hands-on sessions, from Boehringer for a lecture at an educational meeting, and from Acility for acting as chair and for a lecture at an educational meeting, all outside the submitted work.

\section{References}

1 Decaluwé $\mathrm{H}$, Dooms C, D'Journo XB, et al. Mediastinal staging by videomediastinoscopy in clinical N1 non-small cell lung cancer: a prospective multicentre study. Eur Respir J 2017; 50: 1701493.

2 Dooms C, Tournoy KG, Schuurbiers O, et al. Endosonography for mediastinal nodal staging of clinical N1 non-small cell lung cancer: a prospective multicenter study. Chest 2014; 147: 209-215.

3 De Leyn P, Dooms C, Kuzdzal J, et al. Revised ESTS guidelines for preoperative mediastinal lymph node staging for non-small-cell lung cancer. Eur J Cardiothorac Surg 2014; 45: 787-798.

4 Postmus PE, Kerr KM, Oudkerk M, et al. Early and locally advanced non-small-cell lung cancer (NSCLC): ESMO Clinical Practice Guidelines for diagnosis, treatment and follow-up. Ann Oncol 2017; 28: Suppl. 4, iv1-iv21.

5 Zielinski M. Transcervical extended mediastinal lymphadenectomy: results of staging in two hundred fifty-six patients with non-small cell lung cancer. J Thor Oncol 2007; 2: 370-372. 